\title{
Estratégias de pesquisa em contextos de diversidade cultural: entrevistas de listagem livre, entrevistas com informantes-chave e grupos focais
}

\author{
Research strategies in the context of cultural \\ diversity: free-listing interviews, interviews \\ with key-informants and focus groups
}

Cláudia Alquati BISOL'

\begin{abstract}
Resumo
O objetivo deste trabalho é apresentar e discutir três estratégias de pesquisa que, utilizadas separadamente ou combinadas em estudos qualitativos ou quanti-qualitativos, podem facilitar a compreensão do pesquisador acerca do universo cultural dos participantes, especialmente em contextos de diversidade cultural. Entende-se que há diversidade cultural quando a experiência de vida, conceitos, valores e pressupostos teóricos do pesquisador não são os mesmos dos participantes da pesquisa. Os três métodos apresentados, assim como suas possíveis combinações, são: entrevistas de listagem livre, entrevistas com informanteschave e grupos focais. São utilizados exemplos oriundos de pesquisas empíricas no campo da saúde pública e da psicologia para demonstrar a aplicabilidade dessas estratégias.
\end{abstract}

Unitermos: Coleta de dados. Diversidade cultural. Entrevista. Grupos focais.

\begin{abstract}
The objective of this article is to present and discuss three research strategies which, when used separately or combined in qualitative or quanti-qualitative studies, may facilitate the researcher's understanding of the cultural universe of study participants, especially in contexts of cultural diversity. One understands that there is cultural diversity when the life experience, concepts, values and theoretical presuppositions of the researcher are not the same as those of the participants of a study. The three methods that are presented, as well as their possible combinations, are: free-listing interviews, key-informant and focus group interviews. Examples of empirical research in the field of public health and psychology are also presented to demonstrate the applicability of these research strategies.
\end{abstract}

Uniterms: Data collection. Cultural diversity. Interview. Focus groups.

Pesquisas com populações vulneráveis, com grupos especiais e com pessoas de diferentes na- cionalidades geralmente provocam questões importantes e incômodas aos pesquisadores: como acessar

\footnotetext{
$\boldsymbol{\nabla} \boldsymbol{\nabla} \boldsymbol{\nabla}$

1 Universidade de Caxias do Sul, Centro de Ciências Humanas, Curso de Psicologia. R. Francisco Getúlio Vargas, 1130, 95070-560, Caxias do Sul, RS, Brasil. E-mail: <cabisol@ucs.br>.

Apoio: Fogarty International Center (Grant \#R21TW008224) e Universidade de Caxias do Sul.

Agradecimentos: à Dra. Judith Bass (Johns Hopkins Bloomberg School of Public Health), investigadora principal do estudo ao qual este trabalho se vincula.
} 
universos culturais tão diferentes de sua experiência de vida ou do contexto no qual o projeto é concebido, sem comprometer a pesquisa com visões de mundo parciais? A linguagem que se está utilizando faz sentido para os participantes da pesquisa? O que é considerado importante para os pesquisadores, é também relevante no contexto de vida dos participantes? Os pressupostos teóricos que sustentam suas escolhas e análises são adequadas ao contexto cultural específico em que se realiza a pesquisa?

Questões desse tipo têm relação com a sensibilidade do pesquisador às características raciais e étnicas, e também às experiências, normas, valores e padrões de comportamento dos participantes. Têm ainda relação com o desejo desses pesquisadores de incorporar à atividade de pesquisa conhecimentos sobre o contexto histórico, ambiental e sobre as forças sociais que fazem parte da vida do grupo pesquisado (Delva, Allen-Meares \&Momper, 2010). Em outras palavras, trata-se do cuidado com a diversidade cultural ao longo do processo de uma pesquisa.

Este trabalho tem como objetivo apresentar e discutir estratégias de pesquisa que facilitam ao pesquisador a compreensão do universo cultural do grupo pesquisado e promovem uma participação mais ativa dos participantes no processo de pesquisa como um todo. Após se discutir a questão da pesquisa em contextos de diversidade cultural, serão apresentados e discutidos três métodos que podem ser utilizados com relativa facilidade, especialmente quando os pesquisadores se debruçam sobre objetos complexos e multifacetados, como os que caracterizam as pesquisas na área da saúde. Os métodos são: entrevistas de listagem livre, entrevistas com informantes-chave e grupos focais. Essas três estratégias podem ser usadas separadamente ou em conjunto, tanto em estudos qualitativos quanto naqueles que combinam métodos qualitativos e quantitativos. Ao final deste artigo, o uso combinado desses métodos será exemplificado e discutido.

\section{Pesquisa e diversidade cultural}

De uma forma ampla, a diversidade cultural pode ser compreendida como as manifestações de originalidade e pluralidade de identidades que caracterizam os grupos e as sociedades que compõem a humanidade
(Organização das Nações Unidas para Educação, Ciência e a Cultura, 2002). Sendo assim, em se tratando de pesquisa, haverá diversidade cultural toda vez que a experiência de vida, os conceitos e os valores do pesquisador não forem os mesmos dos participantes.

Diferenças linguísticas, culturais e socioeconômicas são bastante óbvias em pesquisas internacionais, cujos pesquisadores são geralmente oriundos de países desenvolvidos, e os dados coletados em países em desenvolvimento. Também é fácil reconhecer a diversidade cultural quando os participantes pertencem a minorias étnicas expressivas dentro de um mesmo país, como em pesquisas com um grupo específico de imigrantes ou com povos indígenas.

Entretanto, é importante lembrar que na grande maioria das pesquisas os pesquisadores são pessoas privilegiadas, enquanto a maior parte dos participantes pertence a camadas vulneráveis da população (Benatar, 2002). Em função disso, é de se esperar que quase sempre existam diferenças entre os pontos de vista e pressupostos teóricos dos pesquisadores, e a visão de mundo dos participantes. Fonseca (2005, p.57) enfatiza que "as condições objetivas de vida levam as pessoas a olharem para o mundo de um ângulo ou de outro". Tendo isso em vista, será importante para o pesquisador tomar consciência de seu contexto cultural específico, reconhecer as limitações de sua visão de mundo, perceber seus preconceitos e colocar-se em posição de aprendiz. Trata-se de desenvolver uma atitude etnográfica que permita mergulhar no contexto cultural dos participantes, com a disposição de aprender com eles sobre seu modo de vida e considerá-los como um "outro" em suas dimensões sociais e culturais, de modo que se possa conhecer a lógica e os desdobramentos de situações que inicialmente poderiam parecer fragmentadas ou incoerentes (Fontoura, 2007).

Para incluir a questão da diversidade e do respeito às diferenças em pesquisa, é necessário não projetar os próprios valores, assim como evitar julgar o comportamento de um participante (Marshall \& Batten, 2003). As diferenças deverão ser discutidas de um modo aberto e com rigor acadêmico (Benatar, 2002). Esses princípios parecem muito claros e até mesmo óbvios, porém são difíceis de serem colocados em prática porque muitas vezes envolvem diferenças sutis que podem facilmente passar despercebidas. Por exemplo, Fonseca (2005) relata 
a tendência de descrever as práticas e maneiras de pensar de grupos populares em termos negativos, de falta e de carência. Segundo essa antropóloga, fala-se de "maternidade assistida" para os ricos e de "controle de natalidade" para as classes populares. Fala-se para o primeiro grupo em "produção independente" e, para o segundo, em "mãe solteira". Fala-se em "família recomposta" para os ricos e "família desestruturada" para as classes populares, quando a organização familiar engendra divórcio e recasamento. Um olhar mais cuidadoso deveria reconhecer que a própria noção de família varia conforme a categoria social e que práticas diferentes não podem ser automaticamente classificadas como ruins.

A pesquisa em contextos de diversidade cultural levanta uma série de questões importantes para os pesquisadores. De acordo com Hines (1993), é indispensável conhecimento a respeito dos problemas e preocupações dessas populações e uso de métodos cuidadosos para que a informação coletada seja relevante e acurada. Hines (1993) distingue ainda três problemas comuns de serem vistos em pesquisas em contextos de diversidade cultural: problemas relativos à equivalência conceitual e linguística, problemas com medidas e problemas com o processo de coleta de dados. A equivalência linguística refere-se à tradução apropriada dos termos, enquanto por equivalência conceitual entende-se que nem sempre os grupos compartilham os mesmos conceitos ou atribuem os mesmos significados aos mesmos fenômenos. Em relação às medidas, indicadores diferentes podem ser necessários para medir o mesmo conceito em diferentes grupos culturais. Também certos conceitos podem divergir em termos de importância para os participantes, e alguns tópicos podem ser tabus em algumas culturas. Em relação ao processo de coleta de dados, os participantes podem ter dificuldades com as dimensões socioculturais das entrevistas e questionários: os maiores problemas se devem a medo, estigma e desconforto que levam a esconder ou distorcer informação, falta de motivação para reconstruir as experiências vividas ou dificuldades de compreensão.

Os métodos que serão apresentados a seguir (entrevistas de listagem livre, entrevistas com informantes-chave e grupos focais) têm em comum algumas características importantes: a) são utilizados em pesquisas em diversas áreas do conhecimento, especialmente em pesquisas com forte preocupação etnográfica (a área da saúde é um exemplo nesse sentido, pois investigações referentes ao adoecimento físico e mental, medicamentos e serviços de saúde têm utilizado métodos como esses); b) podem ser utilizados separadamente ou combinados entre si, como diferentes etapas de um mesmo estudo ou ainda em combinação com outros métodos, como para a construção, adaptação ou aprimoramento de questionários e escalas; c) por serem métodos abertos, permitem aos participantes se expressarem livremente, sem induzir à utilização de determinados termos e sem conduzir a ideias ou conceitos ligados a eventuais interesses prévios do pesquisador; e d) podem ser analisados utilizando-se diferentes métodos de análise de dados.

\section{Entrevistas de listagem livre}

A técnica de listagem livre é geralmente utilizada para identificar domínios culturais ou itens em um domínio cultural, ou seja, percepções e conceitos compartilhados (e não preferências pessoais). Na análise dos resultados pode-se calcular sua importância, proeminência, familiaridade ou representatividade psicológica ou cultural. Pode ser usada também para medir características cognitivas dos informantes, tais como seus padrões de conhecimento sobre um domínio e seus padrões de caracterização (Borgatti, 1998; Ryan, Nolan \& Yoder, 2000). As entrevistas de listagem livre têm sido cada vez mais utilizadas na pesquisa médica para avaliar o conhecimento das pessoas a respeito de sinais e sintomas de certas doenças (Bayliss, Steiner, Fernald, Crane \&Main, 2003).

Listagens livres são geralmente obtidas através de entrevistas semiestruturadas, com uma média de 30 informantes (Borgatti, 1998). O procedimento consiste em perguntar a cada participante uma questão relativa a um domínio de interesse. O participante é então convidado a fornecer uma 'lista' de respostas que representem elementos nesse domínio. Por exemplo, Bayliss et al. (2003), com o objetivo de identificar as barreiras percebidas por pacientes com doenças crônicas (comorbidade) para o autocuidado, solicitaram a eles que listassem todas as coisas que afetam sua habilidade de lidar com suas condições médicas. Durante as entrevistas, os pesquisadores utilizaram ainda duas questões complementares, como facilitadoras: "o que facilita ou 
dificulta os seus cuidados médicos"e "o que você gostaria que mudasse a respeito do que você precisa fazer em função das suas condições médicas". Bolton (2001), por sua vez, utilizou a listagem livre como o primeiro de três métodos etnográficos para investigar como a população de Ruanda percebia os efeitos do genocídio de 1994 em sua saúde mental. As listagens livres foram utilizadas para obtenção de termos locais para sintomas e desordens mentais.

As respostas às entrevistas de listagem livre podem ser analisadas a partir de um enfoque semiquantitativo que permite avaliar padrões de respostas. Borgatti (1998) elenca várias possibilidades de análise: a) contar o número de vezes que cada item é mencionado, organizando-os em ordem decrescente; b) organizar os dados em uma matriz na qual as linhas são os informantes e as colunas os itens; cada célula é categorizada através dos números 1 ou zero (correspondente à menção ou não do item), sendo que a soma por coluna da matriz informa a frequência dos itens, a média das colunas informa a proporção de informantes que menciona cada item, e a soma das linhas informa o número de itens na listagem livre de cada pessoa; c) analisar o número de itens em uma lista, o que reflete a familiaridade da pessoa com o domínio; d) mapear os itens pela coocorrência de itens listados, o que permite identificar aqueles mais frequentemente mencionados em um domínio, ou seja, os itens centrais àquele domínio; e) atentar para a ordem em que os itens são citados, pois os itens mais centrais tendem a ocorrer primeiro e os itens que possuem alguma relação entre si tendem a ser mencionados próximos um ao outro (semelhança cognitiva). Ou ainda, as entrevistas de listagem livre podem conduzir a um uso mais informal dos dados quando se pede aos informantes que deem exemplos de uma categoria conceitual. A análise dos dados coletados nas entrevistas, portanto, pode ter um caráter semiquantitativo ou qualitativo, dependendo do foco de interesse do pesquisador e da fase do processo de pesquisa em que esse método está sendo utilizado, em combinação com outras formas de coleta de dados.

\section{Entrevistas com informantes-chave}

Entrevistas com informantes-chave são comu722 mente utilizadas em pesquisas de campo, na pers- pectiva da etnografia. Nessa perspectiva, campo é entendido como um espaço social e físico cujas fronteiras são definidas em termos de instituições e pessoas ou atividades de interesse em um determinado espaço geográfico (Schensul, 2004).

É comum que os pesquisadores realizem entrevistas semiestruturadas ou abertas, de profundidade. Os entrevistados são indivíduos bem informados, ou seja, pessoas com amplos contatos e envolvimento ativo na comunidade, ou pessoas que têm um conhecimento especial, informação profunda e ampla sobre um sistema, serviço ou outro assunto de interesse específico do pesquisador (Schensul, 2004). As entrevistas permitem obter informações profundas, insights e explicações úteis sobre como os membros de uma comunidade pensam a respeito de determinados eventos, assuntos, pessoas, modos de pensar ou de agir. São muito utilizadas para se obterem informações sobre temas sensíveis, tais como saúde mental e sexualidade (Aboud, 1998). Especificamente na área da saúde, as entrevistas com informantes-chave podem ajudar a compreender diferenças culturais nos conceitos de saúde e doença, planejar prestação de serviços e identificar os recursos existentes na comunidade (Gilchrist \& Williams, 1999).

Os informantes-chave frequentemente se tornam uma via de acesso do pesquisador ao grupo pesquisado. Sanchez, Oliveira e Nappo (2004), por exemplo, afirmam que, em sua pesquisa sobre fatores protetores de adolescentes contra o uso de drogas, os informantes-chave funcionaram como pessoas que facilitaram a aproximação dos pesquisadores com os jovens usuários e não usuários de drogas, de baixo nível socioeconômico, que constituíam sua população em estudo. Nesse caso, os informantes foram profissionais da saúde que trabalham na área de abuso de drogas e populações de risco, religiosos, uma ex-traficante (usuária de drogas, moradora da favela) e uma moradora de favela não usuária de drogas.

Por compartilharem vocabulário, conceitos e vivências, e também por seu conhecimento profundo das normas que regem o funcionamento da comunidade investigada, os informantes-chave poderão se tornar colaboradores especiais da pesquisa: poderão ajudar a formular, expandir ou clarificar as interpretações do pesquisador. Segundo Gilchrist eWilliams (1999, p.74), "o informante-chave ajuda a transformar a compreensão 
limitada do pesquisador sobre a cultura em algo com significado para a cultura do próprio pesquisador. Um informante-chave é um tradutor tanto literal quanto figurativamente".

Em se tratando de uma pesquisa que conjugue vários métodos, os informantes-chave podem ser escolhidos ao longo de observações na comunidade ou podem ser indicados por respondentes de entrevistas preliminares ou de entrevistas de listagem livre. Também é possível utilizar a técnica de snow-ball, em que um primeiro informante-chave indica o subsequente, e assim sucessivamente (Aboud, 1998). Quanto ao número de entrevistas a serem realizadas com cada informante, Schensul (2004) aconselha que sejam feitas pelo menos duas. A primeira oferecerá ideias e pontos de partida que poderão ser mais bem explorados nas entrevistas subsequentes. Segundo esse autor, "a qualidade, profundidade e detalhamento da informação está, geralmente, diretamente relacionada à intimidade e confiança que se desenvolve entre o pesquisador e um informante-chave" (Schensul, 2004, p.570). A duração das entrevistas pode variar de 60 a 90 minutos.

\section{Grupos focais}

A utilização de grupos focais em contextos variados de pesquisa vem se tornando mais comum nos últimos anos. O grande valor dos grupos focais está em sua formatação: uma atividade coletiva, dirigida por um moderador ou facilitador que mantém os participantes 'focados' em uma discussão que foi cuidadosamente planejada e que permite explorar um conjunto específico de questões (Owen, 2001). Segundo Krueger e Casey (2009, p.2), "o propósito de conduzir um grupo focal é escutar e coletar informações. É uma forma de compreender melhor como as pessoas se sentem ou o que pensam sobre uma questão, produto ou serviço. Grupos focais são usados para coletar opiniões".

No contexto da pesquisa acadêmica, os grupos focais permitem: a) explorar as características psicossociais e culturais de uma população; b) aprimorar questionários e outros instrumentos de avaliação; c) complementar a coleta de dados em estudos observacionais ou que utilizam entrevistas de profundidade; d) desenvolver intervenções (Carlini-Cotrim, 1996; Kidd \& Parshall, 2000; Westphal, Bógus \& Faria, 1996). Embora com diferentes finalidades, os grupos focais têm uma característica em comum que os diferencia das entrevistas em grupo: a ênfase ao processo de discussão. Enquanto um entrevistador grupal investe em cada membro do grupo esperando ouvir sua opinião individual sobre determinado tema, o moderador de um grupo focal procura facilitar o processo de discussão, pois sua unidade de análise é o próprio grupo (Gondim, 2003). Nessa perspectiva de compreensão do processo dos grupos focais, considera-se que é a interação entre os participantes do grupo, ou seja, a dinâmica das relações que aí se estabelecem, que permite que percepções, atitudes e crenças sejam manifestadas e que insights aconteçam (Carlini-Cotrim, 1996; Nogueira-Martins \& Bógus, 2004; Winslow, Honein \& Elzubeir, 2002). No entanto, é importante ressaltar que, como método de pesquisa, os grupos focais não têm fins terapêuticos; portanto, não caberá ao moderador do grupo explorar os temas emergentes para além daquilo que for estritamente necessário e previamente estabelecido com os participantes.

O grupo focal é, portanto, um tipo especial de grupo em termos de objetivo, tamanho, composição e procedimentos. As discussões devem ser realizadas várias vezes, em grupos compostos por 5 a 10 participantes que possuam características comuns explicitamente relacionadas ao tópico da pesquisa. Dessa maneira, será possível identificar tendências ou padrões, após uma análise criteriosa e sistemática dos dados coletados. Kind (2004) destaca que, idealmente, devem-se organizar pelo menos dois grupos de discussão para cada variável pertinente, ou organizar um número de grupos suficiente para que ocorra saturação do tema. A autora alerta também que a qualidade ou riqueza das discussões é mais importante do que a quantidade de grupos realizada.

As possibilidades de análise dos dados são várias e dependerão do objetivo do estudo e da forma de registro dos dados escolhida (transcrição literal, transcrição resumida, anotações, memória) (Krueger \& Casey, 2009). De acordo com lervolino e Pelicioni (2001), as formas de análise mais comumente vistas são o sumário etnográfico e a codificação de dados, utilizando-se a análise de conteúdo.

Os grupos focais vêm-se constituindo um método importante nas pesquisas em contextos de diver- 
sidade cultural, pois ajudam os pesquisadores a entender como os participantes percebem as suas experiências e quais significados Ihes atribuem. Isso ajuda a evitar, segundo Vogt, D. N. King e L. A. King (2004), a tendência de impor conceitos e percepções que acabariam por levar a interpretações etnocêntricas e, portanto, equivocadas.

\section{Combinação de métodos}

Os métodos acima descritos podem ser combinados em estudos qualitativos ou utilizados em conjunto com métodos quantitativos. Segundo Flick (2009), a combinação de métodos, também chamada de triangulação, foi pensada, a princípio, como estratégia para validar resultados obtidos com métodos individuais, mas atualmente é considerada como uma forma de complementar o conhecimento e superar os potenciais limitados dos métodos tomados individualmente. A combinação de métodos é defendida por vários autores como meio de dispor perspectivas de diferentes disciplinas para a compreensão de fenômenos complexos que não se conformam aos limites territoriais artificialmente construídos entre as áreas de saber (Carlini-Cotrim, 1996), ou como meio de aprimorar a qualidade de pesquisas levando-se em conta a diversidade cultural, pois se facilita o acesso a resultados culturalmente relevantes e acurados (Hines, 1993). A contribuição de diferentes áreas do saber ou a possibilidade de compor diversos olhares sobre um objeto de estudo complexo e multifacetado é importante para pesquisas na área da saúde, até porque, como diz Scliar (2007, p.30):

O conceito de saúde reflete a conjuntura social, econômica, política e cultural. Ou seja: saúde não representa a mesma coisa para todas as pessoas. Dependerá da época, do lugar, da classe social. Dependerá de valores individuais, dependerá de concepções científicas, religiosas, filosóficas.

Um exemplo de combinação de métodos qualitativos e quantitativos pode ser visto em Bisol, Sperb e Moreno-Black (2008). As autoras utilizaram grupos focais com jovens surdos e ouvintes, com o objetivo de aprimorar um questionário que foi aplicado em uma segunda fase da pesquisa, em formato computadorizado e 724 bilíngue. O processo desenvolvido permitiu identificar as diferenças de contexto de vida entre os participantes e os pesquisadores, adequar o vocabulário e o conteúdo das questões às experiências de vida desses jovens e facilitar a linguagem para maior compreensão dos surdos.

Em um delineamento mais complexo, Andrew, Patel e Ramakrishna (2003) realizaram uma pesquisa com o objetivo de gerar informação para desenvolver serviços de saúde especificamente voltados para adolescentes, integrados ao sistema de saúde já existente, na Índia. Para fazer frente à alta complexidade do objeto de estudo, os autores iniciaram com listagens livres. A análise desses dados serviu para identificar os temas principais, que foram então explorados em profundidade nos grupos focais realizados em seguida. Entrevistas de profundidade com informantes-chave foram depois realizadas para clarificar questões que emergiram nos grupos focais. Em uma segunda fase, os autores geraram um questionário, também discutido em grupos focais, para checagem de validade e vocabulário, aplicado posteriormente a estudantes.

Esse tipo de combinação tem-se tornado frequente e pode ser vista também em Bass, Ryder, Lammers, Mukaba e Bolton (2008), pesquisadores interessados na depressão pós-parto, na República Democrática do Congo. Os autores realizaram entrevistas de listagem livre e entrevistas com informantes-chave para adaptar e validar duas escalas de identificação de depressão pós-parto.

Nesses três exemplos, os resultados obtidos em uma fase qualitativa serviram de base para o planejamento da fase quantitativa realizada na sequência do estudo. Mas uma estrutura inversa também pode ser utilizada: Helman e Yogeswaran (2004), interessados em estudar a percepção sobre as doenças da infância e sobre vacinação na região de Turskey, África do Sul, aplicaram primeiramente um questionário semiestruturado a 60 cuidadores de crianças com menos de 5 anos de idade, depois realizaram grupos focais e entrevistas de listagem livre com o intuito de validar os resultados obtidos nos questionários.

As possibilidades de combinação são muitas, dependem do objetivo e do delineamento da pesquisa, do tempo que se tem disponível, das escolhas e preferências dos pesquisadores e das características da população estudada. O importante, segundo Flick (2009), 
é que os métodos devem ser considerados em igualdade de importância quanto às contribuições que podem prestar ao projeto de pesquisa.

\section{Considerações Finais}

Este artigo dedicou-se à apresentação e discussão de três métodos de pesquisa que, entre outras características, têm a possibilidade de serem combinados entre si e com estratégias quantitativas, em diferentes fases ao longo de um projeto de pesquisa.

Uma limitação deste trabalho é que não foram consideradas as críticas frequentes que são feitas à combinação de estratégias qualitativas e quantitativas. Considerou-se que, em primeiro lugar, esta questão extrapola o objetivo do presente artigo. Em segundo lugar, levou-se em consideração o número crescente de pesquisas que estão utilizando combinações de métodos, principalmente na área da saúde, em estudos realizados por equipes interdisciplinares e em estudos em contextos em que a diversidade cultural se coloca como uma questão que não pode ser deixada em segundo plano. Alguns exemplos de estudos desse tipo foram utilizados ao longo deste artigo para demonstrar a aplicabilidade dessas estratégias de pesquisa.

Mesmo assim, vale ressaltar a importância de se olhar com cuidado para as combinações de métodos. É necessário ter clareza quanto às potencialidades e limitações que cada método apresenta e, mais que isso, quanto às sutilezas que envolvem a preparação dos instrumentos e dos pesquisadores para o momento da coleta em si. Ressalta-se que tanto as abordagens quantitativas quanto as qualitativas apresentam pontos positivos e negativos. Será esta análise cuidadosa dos métodos e abordagens que garantirá a coerência dos estudos.

A questão da análise dos dados também merece ser pensada com cuidado por parte dos pesquisadores. Neste trabalho, foi apresentada de modo genérico a possibilidade de se realizarem diferentes tipos de análise, mas a escolha de como sistematizar essa etapa da pesquisa deve ser epistemologicamente coerente com a base teórica utilizada, e deve também atentar aos potenciais e limitações de cada método.

Por fim, ressalta-se a importância de se conhecerem diferentes estratégias de pesquisa que permitam uma maior aproximação dos pesquisadores ao universo dos participantes, especialmente na área da saúde. Essa área tem se mostrado especialmente rica e aberta ao trabalho interdisciplinar e tem se mostrado também profícua e criativa na tentativa de ampliar o conhecimento e buscar respostas para a complexa relação entre os seres humanos, a saúde e a doença.

\section{Referências}

Aboud, F. E. (1998). Health psychology in global perspective. Thousand Oaks, CA: Sage.

Andrew, G., Patel, V., \& Ramakrishna, J. (2003). Sex, studies or strife? What to integrate in adolescent health services. Reproductive Health Matters, 11 (21), 120-129.

Bass, J. K., Ryder, R. W., Lammers, M. C., Mukaba, T. N., \& Bolton, P. A. (2008). Post-partum depression in Kinshasa, Democratic Republic of Congo: validation of a concept using a mixed-methods cross-cultural approach. Tropical Medicine and International Health, 13 (12), 1-9.

Bayliss, E. A., Steiner, J. F., Fernald, D. H., Crane, L. A., \& Main, D. S. (2003). Descriptions of barriers to self-care by persons with comorbid chronic diseases. Annals of Family Medicine, 1 (1), 15-21.

Benatar, S. R. (2002). Reflections and recommendations on research ethics in developing countries. Social Science \& Medicine, 54 (7), 1131-1141.

Bisol, C. A., Sperb, T. M., \& Moreno-Black, G. (2008). Focus groups with deaf and hearing youths in Brazil: improving a questionnaire on sexual behavior and HIV/AIDS. Qualitative Health Research, 18 (4), 565-578.

Bolton, P. (2001). Local perceptions of the mental health effects of the Rwandan genocide. The Journal of Nervous and Mental Disease, 189 (4), 243-248.

Borgatti, S. (1998). Elicitation techniques for cultural domain analysis. In M.D. LeCompte \& J. J. Schensul (Eds.), Designing and conducting ethnographic research (Ethnographer's toolkit, pp.1-26). Walnut Creek, CA: Alta Mira Press.

Carlini-Cotrim, B. (1996). Potencialidades da técnica qualitativa grupo focal em investigações sobre abuso de substâncias. Revista de Saúde Pública, 30 (3), 285-293.

Delva, J., Allen-Meares, P., \& Momper, S. L. (2010). Crosscultural research (Pocket Guides to Social Research Methods). New York, NY: Oxford University Press.

Flick, U. (2009). Introdução à pesquisa qualitative (3a ed.). Porto Alegre: Artmed.

Fonseca, C. (2005). Concepções de família e práticas de intervenção: uma contribuição antropológica. Saúde e Sociedade, 14 (2), 50-59.

Fontoura, H. A. (2007). A etnografia na saúde: tecendo perspectivas interdisciplinares. Revista SOCERJ, 20 (4), 309-312.

Gilchrist, V., \&Williams, R. (1999). Key informant interviewing. In B. Crabtree \& W. L. Miller (Eds.), Doing qualitative 
research (2 ${ }^{\text {nd }}$ ed., pp.71-78). Thousand Oaks: Sage Publications.

Gondim, S. M. G. (2003). Grupos focais como técnica de investigação qualitativa: desafios metodológicos. Paidéia, $12(24), 149-161$.

Helman, C. G., \& Yogeswaran, P. (2004). Perceptions of childhood immunisations in rural Transkei: a qualitative study. South African Medical Journal, 94 (10), 835-838.

Hines, A. M. (1993). Linking qualitative and quantitative methods in cross-cultural survey research: techniques from cognitive science. American Journal of Community Psychology, 2 (6), 729-746.

lervolino, A. S., \& Pelicioni, M. C. F. (2001). A utilização do grupo focal como metodologia qualitativa na promoção da saúde. Revista da Escola de Enfermagem da USP, 35 (2), $115-21$.

Kidd, P. S., \& Parshall, M. B. (2000). Getting the focus and the group: Enhancing analytical rigor in focus group research. Qualitative Health Research, 10 (3), 293-308.

Kind, L. (2004). Notas para o trabalho com a técnica de grupos focais. Psicologia em Revista, 10 (15), 124-136.

Krueger, R. A., \& Casey, M. A. (2009). Focus groups: a practical guide for applied research ( $4^{\text {th }}$ ed.). Thousand Oaks: Sage Publications.

Marshall, A., \& Batten, S. (2003). Ethical issues in crosscultural research. In W.-M. Roth (Ed.), Connections '03 (pp.139-151). Retrieved on May 12, 2010, from <http:// ejournal.narotama.ac.id/files/EthicallssuesinCrossCulturalResearch.pdf>.

Nogueira-Martins, M. C. F., \& Bógus, C. M. (2004). Considerações sobre a metodologia qualitativa como recurso para o estudo das ações de humanização em saúde. Saúde e Sociedade, 13 (3), 44-57.
Organização das Nações Unidades para Educação, Ciência e a Cultura. (2002). Declaração Universal sobre a Diversidade Cultural. Brasília: UNESCO. Recuperado em março 21, 2009, disponível em <http://unesdoc.unesco.org/images/0012/ 001271/127160por.pdf >.

Owen, S. (2001). The practical, methodological and ethical dilemmas of conducting focus groups with vulnerable clients. Journal of Advanced Nursing, 36 (5), 652-658.

Ryan, G. W., Nolan, J. M., \& Yoder, P. S. (2000). Successive free listing: using multiple lists to generate explanatory models. Field Methods, 12 (2), 83-107.

Sanchez, Z. V. D. M., Oliveira, L. G., \& Nappo, S. A. (2004). Fatores protetores de adolescentes contra o uso de drogas com ênfase na religiosidade. Ciência e Saúde Coletiva, 9 (1) 43-55.

Schensul, J. J. (2004). Key informants. In B. A. Norman (Ed), Encyclopedia of health \& behavior (Vol. 1, pp.569-571). Thousand Oaks: Sage Publications.

Scliar, M. (2007). História do conceito de saúde. PHYSIS: Revista de Saúde Coletiva, 17 (1), 29-41.

Vogt, D. S., King, D. W., \& King, L. A. (2004). Focus groups in psychological assessment: enhancing content validity by consulting members of the target population. Psychological Assessment, 16 (3), 231-243.

Westphal, M. F., Bógus, C. M., \& Faria, M. M. (1996). Grupos focais: experiências precursoras em programas educativos em saúde no Brasil. Boletín de la Oficina Sanitaria Panamericana, 120 (6), 471-482.

Winslow, W. W., Honein, G., \& Elzubeir, M. A. (2002). Seeking Emirati women's voices: the use of focus groups with an Arab population. Qualitative Health Research, 12 (4), 566-575.

Recebido em: 24/5/2011

Aprovado em: 14/5/2012 